\title{
Neural Correlates of Long-Term Object Memory in the Mouse Anterior Cingulate Cortex
}

\author{
Aldis P. Weible, ${ }^{1}$ David C. Rowland, ${ }^{1}$ Caitlin K. Monaghan, ${ }^{2}$ Nicholas T. Wolfgang, ${ }^{1}$ and Clifford G. Kentros ${ }^{1}$ \\ ${ }^{1}$ Institute of Neuroscience, Department of Psychology, University of Oregon, Eugene, Oregon 97403, and ${ }^{2}$ Program in Neuroscience, Boston University, \\ Boston, Massachusetts 02215
}

Damage to the hippocampal formation results in a profound temporally graded retrograde amnesia, implying that it is necessary for memory acquisition but not its long-term storage. It is therefore thought that memories are transferred from the hippocampus to the cortex for long-term storage in a process called systems consolidation (Dudai and Morris, 2000). Where in the cortex this occurs remains an open question. Recent work (Frankland et al., 2005; Vetere et al., 2011) suggests the anterior cingulate cortex (ACC) as a likely candidate area, but there is little direct electrophysiological evidence to support this claim. Previously, we demonstrated objectassociated firing correlates in caudal ACC during tests of recognition memory and described evidence of neuronal responses to where an object had been following a brief delay. However, long-term memory requires evidence of more durable representations. Here we examined the activity of ACC neurons while testing for long-term memory of an absent object. Mice explored two objects in an arena and then were returned $6 \mathrm{~h}$ later with one of the objects removed. Mice continued to explore where the object had been, demonstrating memory for that object. Remarkably, some ACC neurons continued to respond where the object had been, while others developed new responses in the absent object's location. The incidence of absent-object responses by ACC neurons was greatly increased with increased familiarization to the objects, and such responses were still evident 1 month later. These data strongly suggest that the ACC contains neural correlates of consolidated object/place association memory.

\section{Introduction}

Lesions of the hippocampus, as first described in patient HM (Scoville and Milner, 1957), result in a profound anterograde and temporally graded retrograde amnesia. Studies of this phenomenon have contributed to theories of consolidation (Marr, 1970, 1971; Lechner et al., 1999) in which the hippocampus is a temporary repository for information that is subsequently transferred elsewhere for long-term storage (Squire and Alvarez, 1995; Squire and Bayley, 2007). Precisely where this storage occurs is still far from certain. One structure recently implicated in memory consolidation is the anterior cingulate cortex (ACC) (Frankland and Bontempi, 2005). Activity-dependent immediate-early genes are expressed in the ACC during both memory consolidation (Tse et al., 2011) and retrieval (Frankland et al., 2004), and inactivation of the ACC blocks retrieval of both spatial (Teixeira et al., 2006) and nonspatial (Frankland et al., 2004; Ding et al., 2008) remote memory.

While clearly implicating the ACC in memory consolidation and retrieval, these studies do not reveal how such memories are reflected in the spiking activity of ACC neurons. The diversity of functional correlates reported for the rodent ACC complicates

\footnotetext{
Received 0ct. 18, 2011; revised Feb. 23, 2012; accepted Feb. 28, 2012.

Author contributions: A.P.W. and C.G.K. designed research; A.P.W., C.K.M., and N.T.W. performed research;

A.P.W. and D.C.R. analyzed data; A.P.W. and C.G.K. wrote the paper.

This work was supported by Department of Defense Grant W81XWH-07-2-0092.

Correspondence should be addressed to Dr. Aldis P. Weible, Institute of Neuroscience, 225b Huestis Hall, University of Oregon, Eugene, Oregon 97403. E-mail: aldis@uoneuro.uoregon.edu.

DOI:10.1523/JNEUROSCI.5265-11.2012

Copyright $\odot 2012$ the authors $\quad 0270-6474 / 12 / 325598-11 \$ 15.00 / 0$
}

this task (for example, Han et al., 2003; Brecht et al., 2004; Wirtshafter, 2005; Xu et al., 2008). Arguably, the clearest way to demonstrate neural correlates of memory is to observe responses associated with a stimulus that is no longer present. For example, neurons continue to fire following cue removal during working memory tasks. However, the delay period for such experiments is generally only seconds or minutes (Curtis and Lee, 2010). A neural correlate of a consolidated memory should still be present following much greater delays, and should stabilize over time. The present study builds upon our prior work showing clear object/place associations in the firing patterns of ACC neurons (Weible et al., 2009) by examining whether these neurons reflect where a previously explored object had been, and whether greater familiarity stabilizes such correlates.

ACC neurons were recorded as mice explored two objects in a cylindrical arena, and later re-explored the arena with only one of the two original objects remaining. While some mice saw both objects only once, others were familiarized to them over an average of $\sim 2$ weeks. Both groups of mice continued to explore where the object had been, demonstrating memory for that object. Some ACC neurons continued to respond where the object had been, while others developed new responses in that location. Despite the behavioral similarity between groups, absent-object correlates were restricted almost exclusively to familiarized mice. While the 6 h delay between our recordings could simply reveal long-term (i.e., hippocampus-dependent) but not consolidated (i.e., hippocampus-independent) memory, the time course of familiarization was similar to that shown by Vetere et al. (2011) for the expression of consolidation-associated synaptic changes 
in the ACC. Furthermore, absent-object responses following familiarization of a separate group of mice were still evident 1 month later, consistent with remote, consolidated memory. Together, these results suggest that individual ACC neurons show electrophysiological correlates of consolidated object/ place associations.

\section{Materials and Methods}

Experimental overview

The current study involved a modification of traditional recognition memory tasks to test the concept that a neural correlate of memory for a stimulus will most convincingly be demonstrated in the absence of that stimulus. Rodent recognition memory tasks rely on an innate preference for novelty, reflecting the awareness that something in the environment has changed. For example, after having explored a set of objects, rodents spend more time exploring a familiar object that has been moved to a new location (novel location recognition) or a new object that has replaced a familiar one (novel object recognition). Here we examined behavioral and neuronal responses to the absence of one of two previously explored objects, a test that may be referred to as absent-object recognition.

In the first experiment, two groups of mice explored a pair of objects in a cylindrical arena. These mice were then returned to the arena $6 \mathrm{~h}$ later with only one of the two original objects remaining. For one group of mice, the session preceding the $6 \mathrm{~h}$ delay was the only exposure to both objects. Data from these single exposure (S-Exp) mice were compared with data from repeated exposure (R-Exp) mice that were first extensively familiarized to both objects in the arena for many days. The time course of familiarization was sufficient to allow for changes in spine density in ACC neurons associated with memory consolidation (Vetere et al., 2011). Therefore, though the response of both groups to object removal was assessed following the same $6 \mathrm{~h}$ delay, behavioral and neuronal correlates to object removal were expected to reflect consolidated (R-Exp mice) versus nonconsolidated (S-Exp mice) representations of the environment. Individual ACC neurons stably recorded over the $6 \mathrm{~h}$ delay were analyzed to determine how the activity of these cells changed in response to object removal. In this way, we were able to differentiate four different response types: maintained-to-present, maintained-toabsent, starts-to-present, and starts-to-absent. The critical feature to our experimental design was testing for object memory in the absence of a physically present object. This eliminated the possibility that correlates were associated with incoming sensory input, as in the case of traditional recognition memory tests. There was still, however, the possibility that motor correlates associated with exploratory behaviors could be preserved, even though the object itself was absent. Since mice explore objects with their vibrissae, we used electromyographic recordings from the whisker pad to measure whisking activity as mice from both groups explored the two locations to see whether object correlates simply reflected motor function associated with exploratory whisking. These experiments therefore made it possible to (1) examine how ACC neurons would respond to removal of a previously explored object, (2) test for consolidated versus nonconsolidated correlates of object/place associations, and (3) control for correlates that were driven by sensory input or motor system output related to movement planning or execution.

The $6 \mathrm{~h}$ delay in the above experiment enabled the examination of changes in the spiking activity of the same cells in response to object removal. Of course, absent-object correlates following a 6 h delay could be maintained by long-term memory processes, and need not necessarily invoke the consolidation hypothesis. To confirm whether the correlates in the ACC of familiarized mice were the result of a consolidated representation, a second experiment increased the delay period in a separate group of R-Exp mice (R-Exp-30D) from $6 \mathrm{~h}$ to $30 \mathrm{~d}$. This second experiment allowed us to determine whether absent-object correlates following the $6 \mathrm{~h}$ delay were comparable to correlates seen at a delay more generally associated with consolidation and recall of remotely learned information. In this way, we could more confidently judge whether the spiking activity of ACC neurons following familiarization truly reflected consolidated object/place associations.

\section{Animals}

Forty-eight male C57BL6/J mice (Jackson Laboratories) were implanted with an adjustable-depth four-tetrode array for recording ACC neuron activity in vivo during spontaneous exploration of a cylindrical arena and intra-arena objects. All procedures described were performed in accordance with guidelines approved by University of Oregon's Animal Care and Use Committee and the National Institutes of Health Guide for the Care and Use of Laboratory Animals (NIH Publications No. 80-23).

\section{Surgical procedures}

All surgeries were performed using aseptic techniques. Ketamine (100 $\mathrm{mg} / \mathrm{kg})$ was administered as a preanesthetic. Dexamethasone $(0.1 \mathrm{mg} / \mathrm{kg})$ and atropine $(0.03 \mathrm{mg} / \mathrm{kg})$ were administered presurgically to ameliorate possible inflammation and respiratory irregularities, respectively. Surgical anesthesia was maintained with isofluorane (1.25-2.0\%, adjusted as necessary for the appropriate depth of anesthesia). Eyes were kept moist with a thin layer of antibacterial ophthalmic ointment. Mice were positioned in a stereotaxic frame. The skull was then exposed, and lambda and bregma zeroed in the vertical plane. One hole was drilled in the left hemisphere, centered over the caudal anterior cingulate cortex (anteroposterior: $+0.6 \mathrm{~mm}$; ML: $0.4 \mathrm{~mm}$, relative to bregma), through which tetrodes from the recording array were passed (DV: $-1.0 \mathrm{~mm}$, relative to dura). Four additional holes were drilled, two per side $4 \mathrm{~mm}$ lateral from the midline, for the insertion of two stainless steel screws $(00-90 \times$ one-eighth inch) and two ground wires. Grip Cement (Dentsply) was used to secure the array to the screws and skull. Vaseline was applied to isolate the individual tetrodes from the cement, preserving the ability to lower the tetrodes. For the placement of electromyographic (EMG) recording wires, an additional longitudinal incision $5 \mathrm{~mm}$ in length was made in the skin anterior to the array. A 22 ga needle was inserted just beneath the skin of the whisker pad or overlying the maxilla at an upward angle, exiting through the incision. The EMG wires (Teflon-coated, stainless steel wire, $76 \mu \mathrm{m}$ in diameter) were inserted from the top, back through the 22 ga needle. The needle was then retracted, leaving the EMG wire in place beneath the skin, exiting out through the skin. The coating of the exposed wire was stripped, and the wire was then trimmed and allowed to slip back beneath the skin. Mice were administered buprenorphine $(0.06 \mathrm{mg} / \mathrm{kg})$ postoperatively for analgesia to minimize discomfort. All mice were individually housed following surgery and were allowed $7 \mathrm{~d}$ of postoperative recovery.

\section{Behavioral training}

Apparatus. All recording sessions occurred in a cylindrical arena. The cylinder was $60 \mathrm{~cm}$ in diameter and $45 \mathrm{~cm}$ in height, painted black with white geometric shapes stenciled on the inner surface, and was surrounded by an uncued ceiling-height black curtain. Four equally spaced lights directly above the cylinder provided illumination. All entries into the cylinder were from the same position (south). All tracking and neuronal data were recorded for each session as described below in Materials and Methods (Single-neuron recording). All sessions were $30 \mathrm{~min}$ in duration. Between sessions, the mouse was transferred from the cylinder to the home cage set in an opaque transport box. Brown butcher block paper beneath the cylinder was discarded, and the cement floor beneath wiped down with $90 \% \mathrm{EtOH}$. The cylinder was similarly wiped down, and then replaced in the original position and orientation upon a fresh sheet of paper. Objects, when present, were also wiped down between sessions. This process of cleaning the environment and the objects was $\sim 5 \mathrm{~min}$ in duration. Objects were fixed firmly to the paper with a dualsided strip of carpet tape. The objects used were small enough to be easily climbed upon (on average, length, $9.4 \mathrm{~cm}$; width, $5.1 \mathrm{~cm}$; height, $3.8 \mathrm{~cm}$ ) and were identical to those used in our previous study (Weible et al., 2009). Mice were never exposed to objects other than those used in the first exploratory session, as described below.

Behavioral protocol. Mice were separated into one of two groups and run through protocols involving extensive familiarization (R-Exp) or only one session (S-Exp) with both objects. The sequence of recording sessions for both groups is illustrated in Figure 1. All mice explored the empty cylinder [open field (OF)] for two sessions (S1 and S2), followed by a third session [S3, initial exposure (IE)] with two different objects. 
R-Exp mice were returned to the cylinder with both objects 5 min later (S4), followed by an additional six daily familiarization sessions. Six hours following the final familiarization session (S10), R-Exp mice were returned to the cylinder with only one of the two original objects remaining. In contrast, S-Exp mice were placed back in their home cage following initial exposure (S3) and returned to the colony room. Six hours later, these mice were returned to the cylinder with only one of the two original objects remaining. The object that was removed was counterbalanced across animals. Therefore, both groups of mice were tested for their behavioral recognition of the absentobject location following a $6 \mathrm{~h}$ delay. The only difference between groups was the degree of familiarity with both objects before object removal: one session for S-Exp mice versus eight sessions across a number of days for R-Exp mice.

The behavior and spiking activity of a third group of mice (R-Exp-30D) was examined for evidence of consolidated object/place associations. Following the final familiarization session (S10), R-Exp-30D mice were returned to the colony for $30 \mathrm{~d}$. Following this delay, mice were screened for cells (see Single-neuron recording). When clusterable spiking activity was observed, mice were placed back in the cylinder with only one of the two original objects remaining.

\section{Single-neuron recording}

Manufacture of the tetrode recording array was adapted from methods described by Gray et al. (1995). Briefly, four lengths of $18 \mu \mathrm{m}$ diameter $10 \%$ iridium/platinum wire (California Fine Wire) were spun together and heated to fuse the polyamide coating at one end. The coating on the free ends of each wire was removed, and each uncoated wire segment was inserted into a channel of an EIB-16 electrode interface board (Neuralynx) and fixed into place with a gold-coated pin. Each 16-channel EIB16 , loaded with three tetrodes and four EMG wires, was fixed to a Teflon stage mounted on three drive screws. The drive screws $(0-80 \times$ threeeighths inch) provided adjustability of depth for the array as well as the structure between the array and the skull.

Screening. Before the start of training, mice were screened for units in the home cage. A tethered HS-16 operational amplifier (Neuralynx) was plugged into the EIB-16 for monitoring/recording neuronal activity and providing spatial tracking information. If activity of sufficient quality was observed, subjectively defined as the presence of separable, suprathreshold waveform clusters (see Data analysis), data collection would begin. If the neuronal activity observed was of insufficient quality, the array was moved down $\sim 45 \mu \mathrm{m}$, and the animal was returned to the colony room.

Data acquisition. Neuronal data were acquired using a 24-channel Cheetah system (Neuralynx). Neuronal signals were buffered through the HS-16, passed via a $2 \mathrm{~m}$ tether to the ceiling of the recording chamber. In an adjacent room, the signal was amplified and captured using Neuralynx data acquisition software. Thresholds were set such that only waveforms of a specified minimum voltage were stored. A digital camera fixed to the ceiling of the recording chamber linked to the Cheetah system enabled the recording of the animal's position during the course of each session by tracking two LEDs fixed to the HS-16. If units in R-Exp mice were lost before the start of, or during, one of the familiarization sessions (S4-S9), the tetrode array was moved down following completion of the session. Following days in which the tetrodes were moved, animals were rescreened in the home cage before being placed in the cylinder, and the array moved downward again if acceptable activity was not observed. While this process did in some cases add to the total number of days required for an experiment to be completed, the total number of daily recording sessions did not vary between R-Exp mice.

\section{Data analysis}

The activity of individual neurons was isolated off-line using the MATLAB (The MathWorks)-based spike sorting software MClust (A. D. Redish, University of Minnesota, Minneapolis, MN). Pairs of waveform measures were plotted to form clusters of points corresponding to waveforms of individual neurons. The axes of cluster space used were spike height, valley, and energy. MClust provides the flexibility to apply cluster boundaries across multiple recording sessions. Neurons were judged to be "stable" (the same from one session to the next) if similar cluster boundaries could be applied across consecutive sessions without losing cluster separation on at least one pair of axes. Our primary interest lay in determining how the same R-Exp and S-Exp neurons responded across sessions. Therefore, only those neurons found to be stable across the first three sessions (open field 1 and 2 and initial exposure) or the final two sessions (across the $6 \mathrm{~h}$ delay preceding object removal) were analyzed. Recordings from any given tetrode that were not stable across one or both of these blocks of sessions were excluded from further analysis. The R-Exp-30D test used a $30 \mathrm{~d}$ delay between the final familiarization session and the object removal session. Because of the low likelihood of maintaining recordings of the same cell across such a long delay, the criterion of cross-session recording stability was removed, and cells were analyzed solely for responsiveness in either the present and/or absent-object locations.

Rate maps of all behavioral and neuronal data were generated using custom MATLAB routines. Data corresponding to the $60-\mathrm{cm}$-diameter cylinder were parsed into a $2 \mathrm{D}$ matrix of $2 \times 2 \mathrm{~cm}$ pixels. For the purpose of statistical analysis, each object location was assigned a footprint of 64 $\mathrm{cm}^{2}$. Paired $t$ tests were performed using data from visited pixels for each object area and a randomly selected matching number of pixels from the rest of the cylinder, excluding object locations. Analyses of group behavioral data were performed using $t$ tests and mean occupancy time per pixel.

Cells were considered "object-responsive" when such paired $t$ tests yielded $p$ values $<0.05$. Within-group comparisons of counts of respon- 
sive cells were performed using paired $t$ tests on a per animal basis, yielding an absolute measure of responsive cells per animal. Betweengroup comparisons were performed with unpaired $t$ tests on the proportions of responsive cells per animal. For both comparisons, results are illustrated in figures as a proportion (i.e., percentage) of total cells/animal for simplicity. Analyses of group neuronal data were performed with $z$-scores calculated from the pixels used in $t$ tests. $Z$-scores were calculated separately for each object location, with $z=(m-\mu) /\left(\mathrm{SD} / n^{1 / 2}\right)$, where $m$ is the object mean, $\mu$ is the baseline mean, SD is the baseline SD, and $n$ is the sample size. Because responses could come in the form of either significant increases or decreases in firing rate compared with baseline, the absolute value of the $z$-score was used for group comparisons. Statistical analyses of behavior and location-specific spiking activity were performed on unsmoothed data. For illustrative purposes, all behavioral occupancy and neuronal rate maps were smoothed using a $3 \times$ 3 Gaussian kernel, where the weights of the smoothing kernel were from a rotationally symmetric Gaussian filter that was 3 bins wide and with a SD of 1 .

Two additional sets of analyses were performed on neuronal data collected during the two open-field sessions. First, analyses were performed to determine whether ACC neurons exhibited "place fields" analogous to those first observed by O'Keefe and Dostrovsky (1971). This was done to address how specific the restricted fields we see during object exploration are to object/place associations, rather than simply a conjunction of innate spatial correlates with proximal cues (Rivard et al., 2004). Measures included coherence, sparsity, and spatial information, and were calculated with smoothed data. Coherence was calculated as the $z$-transform of the correlation coefficient between the cell's firing rate in a given pixel and the mean in the eight closest pixels; spatial information was calculated as $\Sigma p_{i}\left(\lambda_{i} / \lambda\right) \log _{2}\left(\lambda_{i} / \lambda\right)$, where $i$ is the bin number, $P_{i}$ is the probability for occupancy of bin $i, \lambda_{i}$ is the mean firing rate for bin $i$, and $\lambda$ is the overall mean firing rate; sparsity was calculated as $\lambda^{2} /\left(\Sigma p_{i} \lambda^{2}{ }_{i}\right)$ (Muller and Kubie, 1987; Markus et al., 1994; Fyhn et al., 2004). To test for stability across the two open-field exploration sessions, correlation scores were calculated by converting rate maps into two columns of data points and calculating the correlation coefficient (Pearson's linear correlation). Mismatched pixels between the two columns (resulting from nonvisited pixels in one or the other session) were eliminated from the similarity score calculation, as were common zeros. These measures of spatial activity were also collected from an archived set of hippocampus CA1 pyramidal neurons recorded in a similar environment in the same space. Comparisons of CA1 and ACC neurons for each of these measures across a pair of consecutive $30 \mathrm{~min}$ open-field sessions were performed with ANOVAs. Second, the rate maps were divided into three regions: a central zone 6 pixels in radius ( $\sim 113$ pixels), an intermediate ring extending from 7 to 10 pixels from the center ( $\sim 201$ pixels), and an outer ring 3 pixels in width $(\sim 217$ pixels). Unpaired $t$ tests were performed to compare firing rates among the three regions. These analyses were performed on data collected during the two $30 \mathrm{~min}$ open-field sessions to identify quantifiably classes of neurons that fire preferentially in the center of the arena (bulls-eye cells) or around its periphery (annulus cells). Analysis of mean firing rate between regions was performed with unpaired $t$ tests.

EMG data were acquired continuously at $30 \mathrm{kHz}$ using the same Neuralynx system used for tracking and spiking data. The data were down sampled off-line by a factor of 10, and filtered either for exploratory whisking $(4-12 \mathrm{~Hz})$ or foveal whisking $(12-25 \mathrm{~Hz})$. The instantaneous power in the respective frequency bands was calculated using the Hilbert transform, and then associated with the $x$ and $y$ position of the animal using the synched output of the video tracker. Power maps were created using the same spatial binning procedure as the rate maps. The power for each bin was calculated by summing the power in that bin and dividing by total occupancy.

\section{Histology}

Marking lesions for the identification of electrode placement were made by passing DC current $(+5 \mathrm{~V})$ for $3 \mathrm{~s}$ through a wire from each tetrode from which data were recorded. Mice were then given a lethal dose of pentobarbital sodium (Euthasol $150 \mathrm{mg} / \mathrm{kg}$ ) and perfused transcardially

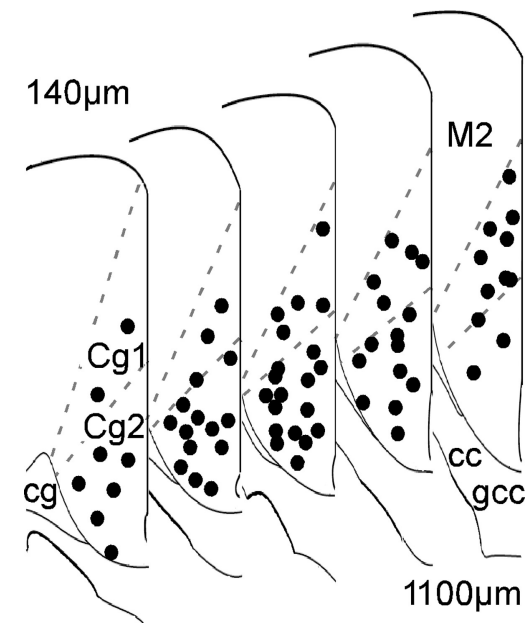

Figure 2. Histological verification of recording sites in caudal anterior cingulate cortex. Tetrode placements extended from the genu of the corpus callosum (gcc) to $\sim 140 \mu$ m anterior to bregma. cc, Corpus callosum; Cg1, Cg2, anterior cingulate gyrus areas 1 and 2, respectively; $c g$, cingulum; M2, secondary motor cortex.

with $0.9 \%$ saline, followed by a $10 \%$ formalin solution. The location of the EMG wires was verified by retracting the skin over the whisker pads and noting the location of each wire, and the brain was extracted and postfixed in $10 \%$ formalin for a minimum of $2 \mathrm{~d}$. Sectioning was performed on a sliding microtome. Every section $(50 \mu \mathrm{m})$ was collected and mounted onto gelatin-coated slides, stained with cresyl violet, and examined under the microscope. Only those data recorded from tetrodes found to be within the caudal ACC, as defined by Jones et al. (2005) and extending from the genu of the corpus callosum to bregma, were included in the present study. Similarly, only EMG data from wires found to be in the whisker pads and overlying the maxilla were analyzed and included.

\section{Results}

The goal of the present study was to determine whether familiarity engenders stable ACC neuronal correlates to where an object had previously been following an extended delay. In the first experiment, the response of single neurons to the removal of one of two objects explored $6 \mathrm{~h}$ earlier was examined in two groups of mice. One group had only had the single earlier session to explore both objects (S-Exp mice), while the other had been extensively familiarized to both objects over the course of many days (R-Exp mice). Only those cells that were stably recorded over the $6 \mathrm{~h}$ delay were analyzed. The second experiment was performed to assess remote memory of absent-object correlates and involved a separate group of familiarized mice that was returned to the cylinder with only one of the two objects remaining following a delay of $30 \mathrm{~d}$. All analyses are based on activity of histologically verified caudal ACC neurons (Fig. 2).

\section{Open field}

Mice were placed in the cylindrical arena for two consecutive sessions to familiarize them to the environment and to determine the spatial specificity of ACC neuron activity. In the hippocampus, highly spatial place fields are characterized by higher coherence, higher spatial information, and lower sparsity. These fields can also remap, following proximal cues placed in the environment (Rivard et al., 2004). It was therefore important to distinguish between object/place associations and spatial correlates that simply remapped to proximal cues. We compared the activity of 281 stably recorded ACC neurons with an archive of 90 CA1 hippocampus pyramidal neurons across the two open-field ses- 


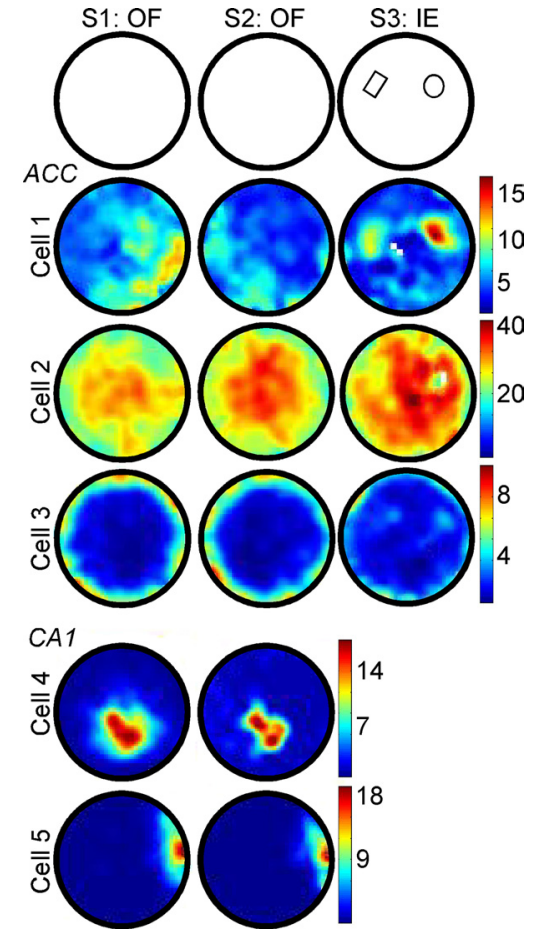

Figure 3. Firing patterns of $\mathrm{ACC}$ neurons in the open field (OF) and upon initial exposure (IE) to objects. The vast majority of anterior cingulate neurons generally did not consistently fire in a specific location across open-field sessions (e.g., Cell 1, S1 vs S2). Exceptions to this were the bulls-eye cells, which reproducibly exhibited peak firing rates near the center of the arena (e.g., Cell 2;22/281 cells), and annulus cells, which fired preferentially around the perimeter (e.g., Cell $3: 11 / 281$ cells). In comparison, CA1 pyramidal neurons typically did fire stably in specific locations in the open field, known as the cell's place field (e.g., Cells 4 and 5). During the session of IE, a large proportion of ACC neurons (136/281 cells; e.g., Cells 1 and 2) did exhibit precise correlates around objects placed in the arena (represented schematically in the top row, S3). Rate maps are autoscaled across sessions. Firing frequency is reported in hertz.

sions. Neurons of the ACC exhibited significantly lower coherence $\left(F_{(1,369)}=23.784, p<0.0001\right.$; ACC: $0.42 \pm 0.02$, SE; CA1: $0.57 \pm 0.04, \mathrm{SE})$ and spatial information $\left(F_{(1,369)}=274.326, p<\right.$ 0.0001 ; ACC: $0.37 \pm 0.03, \mathrm{SE}$; CA1: $1.76 \pm 0.11, \mathrm{SE})$, and significantly higher sparsity $\left(F_{(1,369)}=344.867, p<0.0001\right.$; ACC: $0.74 \pm .014, \mathrm{SE}$; CA1: $0.25 \pm 0.02, \mathrm{SE})$ compared with CA1 pyramidal neurons. Correlation scores across the two open-field sessions were also significantly lower for ACC neurons compared with CA1 pyramidal neurons ( $\mathrm{df}=369, t=5.334, p<0.0001$; ACC: $0.32 \pm 0.02$, SE; CA1: $0.51 \pm 0.03, \mathrm{SE})$. The two most common response types observed from one open-field session to the next were cells with peak firing rates at the center of the arena, which we refer to as bulls-eye cells $(n=22)$, and cells with peak firing rates around the periphery, which we refer to as annulus cells $(n=11)$. These analyses demonstrate that, in general, ACC neurons do not produce classic place fields exhibited by pyramidal neurons of the hippocampus (O'Keefe and Dostrovsky, 1971). Examples of a bulls-eye and annulus cell are illustrated in Figure 3.

\section{Initial exposure}

When objects were first placed in the arena during initial exposure, dwell time around those objects, interpreted as a measure of exploratory behavior, increased significantly compared with the same pixels during the preceding open-field session $(\mathrm{df}=30, t=$ $-4.556, p<0.0001$; open-field session 2: $5.19 \pm 0.13 \mathrm{~s} /$ pixel, SE; initial exposure: $7.75 \pm 0.55 \mathrm{~s} /$ pixel, SE). At the same time, $48.8 \%$
(137/281) of cells responded to one or both objects, with $27.0 \%$ (76/281) exhibiting a significant increase and $21.7 \%(61 / 281)$ exhibiting a significant decrease around one or both objects (only one neuron exhibited an increase to one object and a decrease to another). Most of these responses developed in neurons that had fired diffusely throughout the environment during the preceding open-field session. However, quantifying the incidence of chance responses in object-associated pixels during open-field session 2 first required excluding bulls-eye and annulus cells from both sessions. In the open field, bulls-eye and annulus cells often skewed the results of object/place analyses toward false increases and decreases in response magnitude, respectively. When objects were present, however, bulls-eye cells typically showed a strong reduction in firing rate around object locations (Fig. 3, Cell 2; see also Fig. 5D), while annulus-type responses often exhibited increases in firing in object locations (Fig. 3, Cell 3; see also Figs. 5A, $8 C$ ). To identify these response types in the presence of objects, the pixels associated with the objects were omitted from the analysis. After excluding the responses of putative bulls-eye and annulus cells during the initial exposure session, $38.4 \%$ (108/281) of the remaining cells exhibited object-associated responses. In contrast, exclusion of bulls-eye and annulus response types during open-field session 2 yielded essentially a chance proportion of only $7.1 \%(20 / 281)$ of cells exhibiting a significant response in either location to be subsequently occupied by an object. These data demonstrate that ACC neurons exhibit specific changes in activity during exploration of objects. No differences were found between neurons from mice subsequently divided into S-Exp and $\mathrm{R}$-Exp groups for mean firing rate in the open field $(4.60 \pm 1.18$ and $4.79 \pm 1.0 \mathrm{~Hz} \mathrm{SE}$, respectively) or object-associated response magnitudes (mean $z$-scores of $3.40 \pm 0.20$ and $3.35 \pm 0.29, \mathrm{SE}$, respectively) during initial exposure.

\section{Object removal}

Memory for object/place associations was tested by removing one of two previously explored objects from the environment. This test was performed in two groups of mice distinguished by their relative familiarity with the objects in their locations. S-Exp mice $(n=15)$ had explored both objects only once (initial exposure) before the object removal session. In contrast, R-Exp mice $(n=$ 16) were extensively familiarized to both objects over many days (mean duration, $13.4 \pm 2.1 \mathrm{~d}$ from initial exposure to object removal; range, 7-27 d from initial exposure to object removal). Figure 4 illustrates occupancy from an S-Exp and R-Exp mouse during object removal and the preceding session. Following object removal, both groups of mice continued to explore the absent (R-Exp: $\mathrm{df}=30, t=3.630, p=0.001$; S-Exp: $\mathrm{df}=28, t=2.625$, $p=0.0139)$ and present (R-Exp: $\mathrm{df}=30, t=4.443, p=0.0001$; S-Exp: $\mathrm{df}=28, t=2.975, p=0.006)$ object locations. The time spent exploring the two locations (absent: $\mathrm{df}=29, t=1.463, p=$ 0.1542; R-Exp: $6.94 \pm 0.82$ s/pixel, SE; S-Exp: $5.48 \pm 0.55 \mathrm{~s} /$ pixel, SE; present: $\mathrm{df}=29, t=1.262, p=0.2171$; R-Exp: $8.68 \pm 1.06$ s/pixel, SE; S-Exp: $6.87 \pm 0.96$ s/pixel, SE) did not differ between groups. These data clearly demonstrate the behavioral salience of the absent-object location for both R-Exp and S-Exp mice.

Table 1 summarizes the responses of 291 neurons (R-Exp: 166 neurons; S-Exp: 125 neurons) following object removal. A response that had been seen to a specific object during the previous session is listed as "maintained," while a response first seen during object removal (i.e., had not occurred in that object/location previously) is listed as "starts." Cells that were responsive in both locations were included in both "to present" and "to absent" columns. Thus, four general response types of particular interest 
A

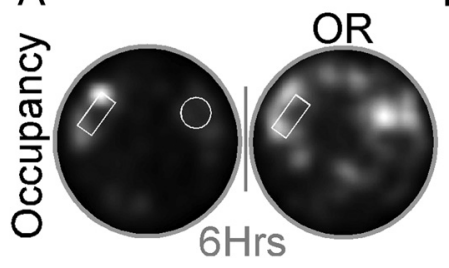

B

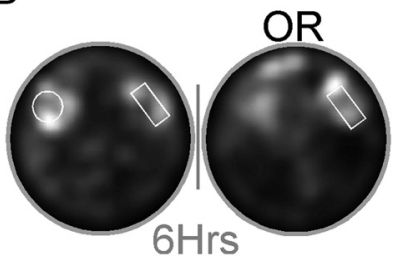

Figure 4. Mice preferentially explored the location of the absent object. Example occupancy maps (average tracker output over time) illustrate exploratory behavior for both present-object (white outlines) as well as the absent-object locations during the object removal (OR) session. Absent-object exploration was seen both for mice that explored the objects only once (single exposure; $\boldsymbol{A}$ ) as well as mice that had been extensively familiarized to both objects before object removal (repeated exposure; $\boldsymbol{B}$ ). See Results (Object removal) for group behavioral data. Gray bars represent the $6 \mathrm{~h}$ delay between sessions. Occupancy scale is the same in both sessions.

Table 1. Neuronal responses at present and absent object locations

\begin{tabular}{llllll}
\hline & \multicolumn{2}{l}{ Repeated exposure } & & Single exposure \\
\cline { 2 - 3 } \cline { 5 - 6 } Ro present & To absent & & To present & To absent \\
Response & {$[29.5 \%(49 / 166)]$} & {$[30.7 \%(51 / 166)]$} & & {$[41.6 \%(52 / 125)]$} & {$[6.4 \%(8 / 125)]$} \\
\hline Maintained & $22 \%$ & $14 \%$ & $22 \%$ & $2 \%$ \\
Starts & $8 \%$ & $17 \%$ & $19 \%$ & $4 \%$ \\
\hline
\end{tabular}

in the present study were possible: maintained-to-present, maintained-to-absent, starts-to-present, and starts-to-absent. Examples of each of these are illustrated in Figure 5. Figure $5 A$ illustrates a maintained-to-present response, demonstrating an example of the null hypothesis that ACC neurons will only fire where an object physically exists. Figure $5 B$ illustrates another maintained-to-present response that also demonstrates object selectivity in the firing of ACC neurons. Figure $5 C-F$ illustrates spiking correlates to where the object used to be. Figure $5 C, D$ illustrates maintained-to-absent responses, and also demonstrate the potential for overlap in the "to present" and "to absent" categories mentioned above (starts-to-present and maintainedto-present, respectively). Figure $5 E$ illustrates a more specific maintained-to-absent response, demonstrating firing that continues specifically to the absent object. Figure $5 F$ illustrates a starts-to-absent response, in which a previously unresponsive cell develops a firing correlate to where the object had been. Finally, Figure $5 G$ illustrates a starts-to-present response, firing to the remaining object only after the other object has been removed. Cells were also observed that ceased responding to the present and/or absent object (data not shown).

As illustrated in Figure 6, how R-Exp and S-Exp neurons responded following the removal of one of two previously explored objects differed in a number of interesting ways. The number of R-Exp neurons responding around the absent object (30.7\%) and the present object $(29.5 \%)$ was essentially identical ( $p=0.9100$; absent: $3.00 \pm 0.54$ cells/mouse, SE; present: $3.06 \pm 0.74$ cells/ mouse, SE) (Fig. 6A). In contrast, far fewer S-Exp neurons responded around the absent object compared with the present object ( $\mathrm{df}=14, t=5.956, p<0.0001$; absent: $0.53 \pm 0.22$ cells/mouse, SE; present: $3.47 \pm 0.45$ cells/mouse, SE) (Fig. $6 \mathrm{~A}$ ). In fact, the proportion of S-Exp cells responding to the absent object (6.4\%; see Table 1) was comparable to "chance" object space responding $(7.1 \%)$ observed as mice explored the open field, as described above in Results (Initial exposure). Between the two groups, absent-object responses were far more prevalent among R-Exp neurons compared with S-Exp neurons ( $\mathrm{df}=29$, $t=4.4, p=0.0001 ; 6.4 \pm 2.4 \%$ cells, SE; R-Exp: $30.7 \pm 4.6 \%$

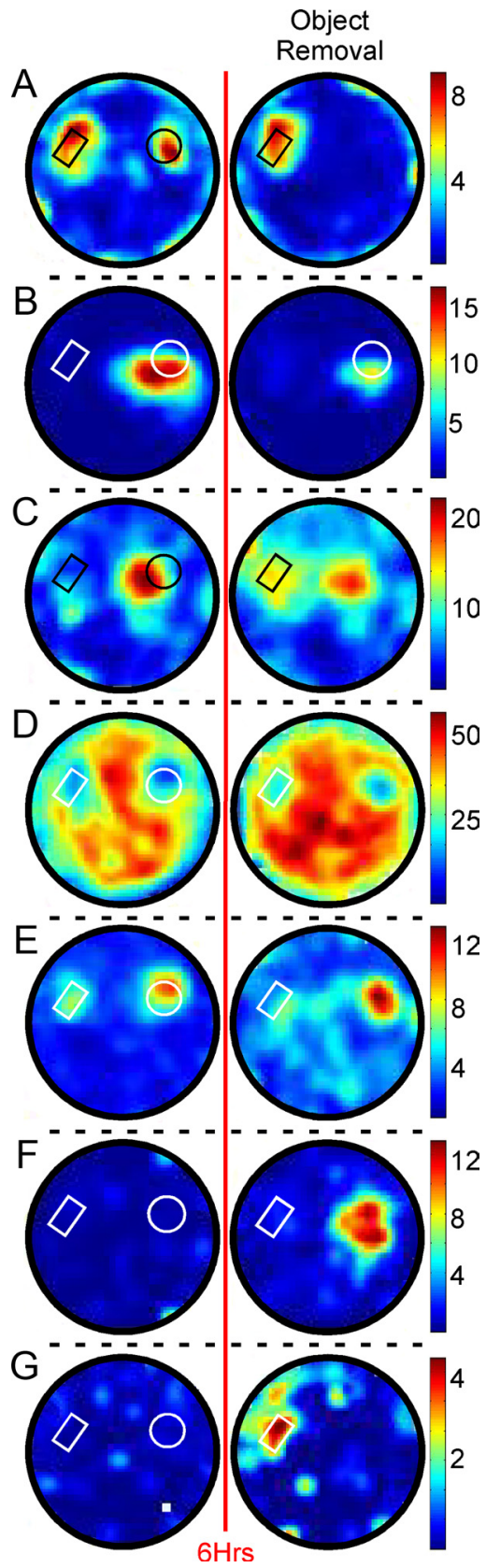

Figure 5. Responses of $A C C$ neurons to object removal. Rate maps of ACC neurons before (first column) and after (second column) removal of one object. $\boldsymbol{A}, \boldsymbol{B}$, Maintained-to-present cells responded only to physically present objects. $\boldsymbol{C}-\boldsymbol{E}$, Maintained-to-absent cells continued to respond where the object had previously been, and could include those cells that began $(\boldsymbol{C}$; starts-to-present), continued ( $\boldsymbol{D}$; maintained-to-present), or ceased $(\boldsymbol{E})$ responding to the remaining object. $\boldsymbol{F}$, Starts-to-absent cells developed a response specifically to the object's absence. G, Starts-to-present cells developed a new response specifically following object removal. Cells $C$ and $D$ and Cells $E$ and $F$ were recorded from two mice. The red line represents the $6 \mathrm{~h}$ delay preceding the object removal session. Black or white outlines illustrate the positions of present objects; line color was varied for contrast. Firing frequency is reported in hertz.

cells, SE) (Fig. 6A). Importantly, group differences extended to the present object. Nearly twice as many S-Exp neurons changed their response to the present object, including starts-to-present responses as well as neurons that ceased responding to the present object following object removal $(\mathrm{df}=29, t=3.4, p=0.002$; S-Exp: $37.7 \pm 4.2 \%$, SE; R-Exp: $20.1 \pm 3.1 \%$, SE). The representation of the environment as a whole also differed between groups. Comparing the rate maps of all neurons (i.e., not just the 
A

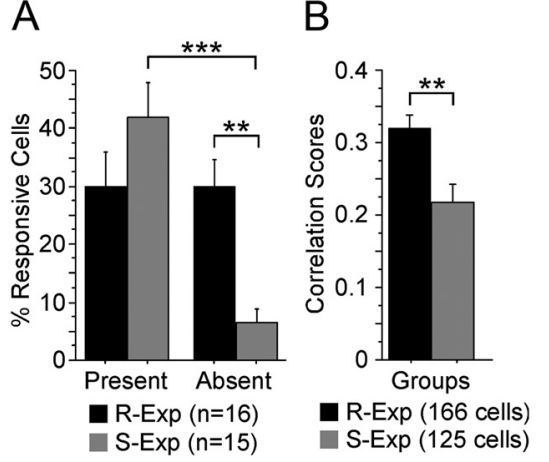

Figure 6. Increased familiarity increased responses to the absent-object location and overall spatial correlations of ACC neurons. $\boldsymbol{A}$, Percentage of neurons from two groups of mice differing in the amount of exposure to the objects (R-Exp and $S$-Exp) that responded to the presentobject versus the absent-object locations during the object removal session. Familiarization to the objects resulted in a similar number of ACC neurons of R-Exp mice responding to the absent object as to the present object, while the ACC neurons of mice that had previously explored both objects only once (S-Exp mice) responded almost exclusively to the present object. $\boldsymbol{B}$, Spatial correlation scores comparing activity for all stably recorded R-Exp (166) and S-Exp (125) neurons before and after object removal show that the entire ACC representation of the environment was more stable when the animals were familiarized to the environment. ${ }^{* *} p<0.001$; ${ }^{* * *} p<0.0001$.

object-selective ones) before and after object removal yielded significantly greater correlation scores for R-Exp neurons than S-Exp neurons $(\mathrm{df}=289, t=3.404, p=0.0008$; S-Exp: $0.22 \pm$ 0.02 , SE; R-Exp: $0.32 \pm 0.02$, SE) (Fig. $6 B$ ). The R-Exp correlation scores comparing rate maps with and without one of the objects were in fact comparable to the correlation scores seen between sessions 3 and 4, which were separated by only a few minutes, and during which both objects were physically present $(p=0.2625$; R-Exp sessions 3 and 4: $0.35 \pm .023, \mathrm{SE})$. The mean magnitude of present-object responses was similar between groups (R-Exp $z$-score: $2.71 \pm 0.24, \mathrm{SE}$; S-Exp $z$-score: $2.49 \pm$ 0.19 , SE). No significant differences between or within groups were observed during the session before object removal in the proportion of neurons responding around either the subsequently removed object (R-Exp: $37 \pm 6 \%$ cells, SE; S-Exp: $47 \pm$ $6 \%$ cells, SE) or the object that remained (R-Exp: $46 \pm 5 \%$ cells, SE; S-Exp: $51 \pm 8 \%$ cells, SE). Nor were any differences observed between or within groups during the session before object removal for mean response magnitude to either the subsequently removed object (R-Exp $z$-score: $2.37 \pm 0.23$; S-Exp $z$-score: $2.30 \pm 0.21$ ) or the object that remained (R-Exp $z$-score: $2.68 \pm$ 0.28 , SE; S-Exp $z$-score: $2.54 \pm 0.19, \mathrm{SE}$ ). The lack of any differences in these measures demonstrates that the group differences during the object removal session were specific to the object's absence and not the result of an unintended bias toward one object/location or another. These data demonstrate far greater stability of the environmental representation across the $6 \mathrm{~h}$ delay in the neurons of extensively familiarized mice, with (1) more R-Exp neurons having absent-object responses, (2) fewer R-Exp neurons changing their response to the present object, and (3) greater correlation scores for R-Exp neurons overall compared with S-Exp neurons.

The response magnitude of neurons could change over time, particularly in the absent-object location. To examine this in greater detail, we analyzed data from the $30 \mathrm{~min}$ object removal session and the session preceding it in three 10 min blocks. The time spent by each group of mice in the absent-object location was comparable across 10 min blocks. Some neurons responded stably over time to the absent object, while others tapered off. However, 37\% (19/51) of absent-object responses developed only after the first $10 \mathrm{~min}$ of the object removal session. This proportion of delayed-onset responses was not seen during the previous session when the object was present $(\mathrm{df}=28, t=2.550$, $p=0.0165$; R-Exp session 10: $13.4 \pm 6.3 \%$ cells, SE; R-Exp object removal: $37 \pm 7.4 \%$ cells, SE). These results indicate that (1) some neurons exhibit a delayed response to the absent object independent of a change in behavior and (2) the high proportion of such responses is specifically associated with the object's absence.

The greater proportion of sustained R-Exp cell responses to the absent object is directly associated with the greater familiarity of these mice with the objects and the environment. It is possible that mice with less familiarity may have responses to the absentobject location that degrade too rapidly to be detected when analyzing the entire $30 \mathrm{~min}$ object removal session. This did not, however, appear to be the case. During the session preceding object removal, approximately one third (44/125) of neurons were responsive to the object that was subsequently removed. When examined during the first 10 min following object removal, only 5 of 44 neurons exhibited a significant response in the absent-object location, of which only 3 responded significantly over the duration of the object removal session (see Table 1, Maintained, to Absent). Similarly, only 6 neurons exhibited a new response in the absent-object location during the first 10 min, 5 of which remained significant over the course of the entire session (see Table 1, Starts, to Absent). These data demonstrate that the lack of absent-object correlates from S-Exp neurons is not simply attributable to a less stable representation in general, and instead confirm that familiarity increases the incidence of absent-object correlates in ACC neurons.

\section{Object exploration and whisker EMG activity}

Group differences in neuronal correlates could be attributable to motor behaviors. Though the object is no longer present, ruling out a strictly sensory explanation for the data, ACC neuron correlates in that location could reflect motor functions associated with exploration. Arguably, the most important exploratory motor function for rodents is whisking. For this reason, in addition to analyzing the time spent exploring the two object locations, we examined EMG activity recorded from the whisker pads. Foveal whisking, characterized by a higher frequency and lower amplitude relative to exploratory whisking, is associated with the active exploration of surfaces (Berg and Kleinfeld, 2003). We analyzed EMG activity in the beta frequency range associated with foveal whisking (12-25 Hz) (Fig. 7A) following object removal to compare EMG activity both around the present and absent objects and between groups, and found significantly higher frequency whisking immediately around the remaining object for both R-Exp mice and S-Exp mice (total: $\mathrm{df}=31, t=4.752, p<0.0001$; present: $17.73 \pm 0.14 \mathrm{~Hz}$, SE; BL: $17.47 \pm 0.13 \mathrm{~Hz}$, SE; R-Exp: $\mathrm{df}=14, t=3.215, p=0.0062$; present: $18.00 \pm 0.21 \mathrm{~Hz}$, SE; BL: $17.74 \pm .15 \mathrm{~Hz}, \mathrm{SE} ; \mathrm{S}$-Exp: $\mathrm{df}=16, t=3.401, p=0.0037$; present: $17.49 \pm 0.19 \mathrm{~Hz}, \mathrm{SE} ; \mathrm{BL}: 17.22 \pm 0.18 \mathrm{~Hz}, \mathrm{SE})$. This was accompanied by a significant reduction in signal amplitude around the remaining object for both R-Exp and S-Exp mice (Fig. 7B; total: $\mathrm{df}=31, t=-5.564, p<0.0001$; present: $24.0 \pm 0.9 \mu \mathrm{V}^{2} / \mathrm{Hz}, \mathrm{SE}$; BL: $26.2 \pm 0.9 \mu \mathrm{V}^{2} / \mathrm{Hz}$, SE; R-Exp: $\mathrm{df}=12, t=-4.132, p=$ 0.0010; present: $26.5 \pm 1.0 \mu \mathrm{V}^{2} / \mathrm{Hz}$, SE; BL: $28.8 \pm 1.0 \mu \mathrm{V}^{2} / \mathrm{Hz}$, SE; S-Exp: $\mathrm{df}=16, t=-3.690, p=0.0020$; present: $21.8 \pm 1.1$ $\mu \mathrm{V}^{2} / \mathrm{Hz}$, SE; BL: $\left.24.0 \pm 1.2 \mu \mathrm{V}^{2} / \mathrm{Hz}, \mathrm{SE}\right)$. Neither whisking frequency nor amplitude differed significantly from baseline or be- 


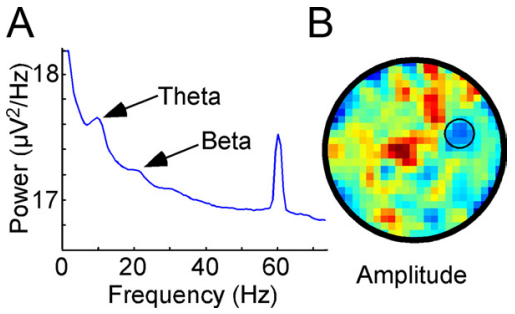

Figure 7. EMG recordings from the whisker pad reflect modulation associated with physically present objects. $A$, Peaks in the power spectrum were observed at frequencies reflecting general whisking (theta) and foveal whisking (beta) associated specifically with object exploration. $\boldsymbol{B}, A$ "whiskmap" plotting EMG amplitude filtered at frequencies associated with foveal whisking (12-25 Hz) reveals a clear decrease around the present object (small black circle). Both $\mathrm{R}$-Exp and S-Exp mice exhibited a significant increase in whisking frequency (R-Exp: $\mathrm{df}=14$, $t=3.215, p=0.0062 ; S$-Exp: $\mathrm{df}=16, t=3.401, p=0.0037$ ) and a significant decrease in whisking amplitude (R-Exp: $\mathrm{df}=12, t=-4.132, p=0.0010 ; \mathrm{S}$-Exp: $\mathrm{df}=16, t=-3.690$, $p=0.0020$ ) around the remaining object when filtering at foveal frequencies. No significant differences occurred for either group around the absent object, however, suggesting that the absent-object correlates were not due to changes in exploratory whisking.

tween groups in the absent-object location (frequency: R-Exp absent: $17.98 \pm 0.24 \mathrm{~Hz}, \mathrm{SE}$; S-Exp absent: $17.22 \pm 0.18 \mathrm{~Hz}, \mathrm{SE}$; amplitude: R-Exp absent: $28.5 \pm 1.2 \mu \mathrm{V}^{2} / \mathrm{Hz}$, SE; S-Exp absent: $\left.24.6 \pm 1.2 \mu \mathrm{V}^{2} / \mathrm{Hz}, \mathrm{SE}\right)$. No significant differences were found around either object location when frequency or amplitude analyses were performed on data recorded simultaneously from separate EMG wires located in the muscle overlying the maxilla (frequency: R-Exp present: $17.73 \pm 0.11 \mathrm{~Hz}, \mathrm{SE}$; R-Exp absent: $17.51 \pm 0.08 \mathrm{~Hz}, \mathrm{SE}$; R-Exp BL: $17.66 \pm 0.1 \mathrm{~Hz}$, SE; S-Exp present: $17.61 \pm 0.14 \mathrm{~Hz}, \mathrm{SE}$; S-Exp absent: $17.53 \pm 0.18 \mathrm{~Hz}, \mathrm{SE}$; S-Exp BL: $17.56 \pm 0.12 \mathrm{~Hz}$, SE; amplitude: R-Exp present: $28.7 \pm$ $0.6 \mu \mathrm{V}^{2} / \mathrm{Hz}$, SE; R-Exp absent: $29.8 \pm 0.9 \mu \mathrm{V}^{2} / \mathrm{Hz}$, SE; R-Exp BL: $29.5 \pm 0.7 \mu \mathrm{V}^{2} / \mathrm{Hz}$, SE; S-Exp present: $25.2 \pm 0.9 \mu \mathrm{V}^{2} / \mathrm{Hz}, \mathrm{SE}$; S-Exp absent: $28.3 \pm 1.5 \mu \mathrm{V}^{2} / \mathrm{Hz}, \mathrm{SE}$; S-Exp BL: $27.1 \pm 1.0$ $\left.\mu \mathrm{V}^{2} / \mathrm{Hz}, \mathrm{SE}\right)$. Similarly, no significant differences were observed around either object location for analyses of frequency or amplitude when filtering at lower frequencies associated with nonfoveal whisking (5-12 Hz) (frequency: R-Exp present: $7.48 \pm 0.10$ Hz, SE; R-Exp absent: $7.58 \pm 0.43 \mathrm{~Hz}$, SE; R-Exp BL: $7.55 \pm 0.10$ $\mathrm{Hz}, \mathrm{SE}$; S-Exp present: $7.04 \pm 0.22 \mathrm{~Hz}$, SE; S-Exp absent: $7.33 \pm$ $0.16 \mathrm{~Hz}, \mathrm{SE}$; S-Exp BL: $7.25 \pm 0.14 \mathrm{~Hz}, \mathrm{SE}$; amplitude: R-Exp present: $28.9 \pm 0.8 \mu \mathrm{V}^{2} / \mathrm{Hz}$, SE; R-Exp absent: $29.6 \pm 0.8 \mu \mathrm{V}^{2} /$ $\mathrm{Hz}, \mathrm{SE}$; R-Exp BL: $29.4 \pm 1.0 \mu \mathrm{V}^{2} / \mathrm{Hz}$, SE; S-Exp present: $26.8 \pm$ $1.0 \mu \mathrm{V}^{2} / \mathrm{Hz}$, SE; S-Exp absent: $28.7 \pm 1.3 \mu \mathrm{V}^{2} / \mathrm{Hz}$, SE; S-Exp BL: $\left.27.6 \pm 1.2 \mu \mathrm{V}^{2} / \mathrm{Hz}, \mathrm{SE}\right)$. The EMG data reveal hallmarks of foveal whisking only around the present object, and that whisking behavior around the present and absent objects did not differ between R-Exp and S-Exp mice. These data indicate that differences in whisking activity do not account for differences in neuronal responding to the absent object between groups.

\section{Object removal: $30 \mathrm{~d}$}

We demonstrated above that familiarization significantly increased the proportion of neurons responding to the absent object following a $6 \mathrm{~h}$ delay. Given that synaptic changes in the ACC associated with memory consolidation occur well within the time course of our familiarization protocol (Vetere et al., 2011), we believe that familiarization enables us to distinguish between consolidated (in R-Exp mice) and nonconsolidated (in S-Exp mice) neuronal representations of object/place associations. If the ACC neuronal correlates to the absent object are in fact associated with these same structural changes, such correlates to the absent-object location should persist for the duration of the be-

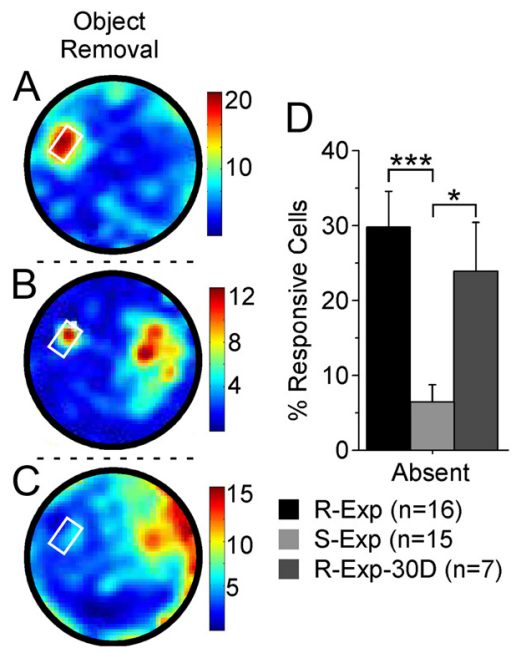

Figure 8. Absent-object responses of ACC neurons following a 30 d delay reflect consolidation of object/place associations. Mice from a third group (R-Exp-30D, $n=7$ ) were familiarized identically to the R-Exp mice, but were then returned to the cylinder following a delay of $30 \mathrm{~d}$ rather than $6 \mathrm{~h}$ after the final familiarization session. Following the $30 \mathrm{~d}$ delay, responses were observed to the present object $(\boldsymbol{A})$, both present and absent objects $(\boldsymbol{B})$, and the absent object alone $(\boldsymbol{C})$. The proportion of R-Exp-30D cells/mouse responding to the absent object $30 \mathrm{~d}$ following the final familiarization session was similar to the proportion of R-Exp neurons, and significantly greater than the proportion of S-Exp neurons, observed responding to the absent object following the $6 \mathrm{~h}$ delay. The remaining object is indicated by the white circle. Firing frequency is reported in hertz. ${ }^{*} p<0.05 ;{ }^{* * *} p<0.0001$.

havioral memory. Here we tested whether these neuronal correlates would be evident a month or more following the last exploration session, matching the timeframe used by Frankland et al. (2004) and Bontempi et al. (1999).

Mice (R-Exp-30D; $n=7$ ) were extensively familiarized to both objects over many days (mean range, $9.6 \pm 1.4 \mathrm{~d}$ from initial exposure to the final familiarization session; range, $7-17 \mathrm{~d}$ from initial exposure to the final familiarization session). Thirty days following the final familiarization session, mice continued to preferentially explore the location in which the object had previously been ( $\mathrm{df}=6, t=3.476, p=0.0132$; absent: $7.02 \pm 0.73$ s/pixel, SE; BL: $4.59 \pm 0.10 \mathrm{~s} /$ pixel, SE). Of the 84 neurons recorded during this session, 24\% (20/84) exhibited correlates to the absent-object location (Fig. 8, Cells B and C). The proportion of R-Exp-30D neurons responding to the absent object was comparable to the proportion of R-Exp neurons ( $p=0.4511$; R-Exp30D: $24 \pm 7 \%$, SE; R-Exp: $31 \pm 5 \%$, SE), and significantly greater than the proportion of S-Exp neurons $(\mathrm{df}=20, t=2.908, p=$ 0.0087; R-Exp-30D: $24 \pm 7 \%$, SE; S-Exp: $6 \pm 2 \%$, SE), responding to the absent object following the $6 \mathrm{~h}$ delay (Fig. $8 \mathrm{D}$ ). The proportion of R-Exp-30D cells responding to the remaining object $(44 \pm 6 \%)$ did not differ significantly with that of R-Exp $(30 \pm 6 \%, p=0.1631)$ or S-Exp $(42 \pm 6 \%, p=0.8113)$ neurons. The onset of some ( $40 \%$ or $8 / 20)$ of these responses was delayed, reaching significance only after the first $10 \mathrm{~min}$, as was seen with R-Exp neurons following the $6 \mathrm{~h}$ delay. The mean amplitude of significant responses in the two locations was comparable for both the entire session $\left(F_{(1,52)}=0.027, p=0.8708\right.$; present $z$-score: $2.71 \pm 0.24$, SE; absent $z$-score: $2.77 \pm 0.36$, $\mathrm{SE})$ as well as across $10 \mathrm{~min}$ bins $\left(F_{(2,104)}=0.406, p=0.6672\right.$. These data demonstrate that ACC neurons of mice familiarized $30 \mathrm{~d}$ earlier respond in the absent-object location similarly to neurons tested following a $6 \mathrm{~h}$ delay, and confirm that our familiarization protocol results in response stability that 
would be expected of remote retrieval of a consolidated representation.

\section{Discussion}

In the present study, we have shown that mice preferentially explore areas where an object had previously been, and that caudal ACC neurons also respond in that location, presumably reflecting the memory of the object/place association. These absentobject responses were largely restricted to animals extensively familiarized to both objects over the course of many days. Remarkably, such absent-object correlates were still evident when a separate group of mice was returned to the arena $30 \mathrm{~d}$, rather than $6 \mathrm{~h}$, following the final familiarization session. Together, these data show that ACC neurons have properties one would expect of memories in the process of consolidation, adding neurophysiological evidence to prior molecular and behavioral work, suggesting a role for the ACC in memory consolidation (Frankland and Bontempi, 2005; Vetere et al., 2011).

Consolidation theory posits that information initially processed in the hippocampus is subsequently stored in cortex (Marr, 1970, 1971; Squire and Alvarez, 1995; Frankland and Bontempi, 2005; Squire and Bayley, 2007). This theory would suggest that electrophysiological correlates of a consolidating cortical representation should stabilize over time. Behaviorally, both R-Exp and S-Exp mice recognized the absent-object location following a $6 \mathrm{~h}$ delay. However, ACC neuronal recognition of the absent-object location was essentially restricted to familiarized $\mathrm{R}$-Exp mice. Some neurons continued to respond to where the object had been (maintained-to-absent responses), while others developed responses to the object's absence (starts-to-absent responses). The neuronal representation of the arena as a whole was also more stable in familiarized mice. Similar absent-object correlates were present in R-Exp-30D mice tested 1 month following familiarization. In contrast, the number of S-Exp neurons responding to the absent object following a single previous exploration session was essentially at chance, and responding to the remaining object following object removal was less stable compared with familiarized mice. Together, these data are entirely consistent with ACC involvement in the gradual process of consolidation (Frankland and Bontempi, 2005). In S-Exp mice, behavioral recognition of the absent-object location was likely supported by a nonconsolidated neuronal representation in the hippocampal formation, as suggested by hippocampal involvement in recognition memory (Hammond et al., 2004; Sarkisyan and Hedlund, 2009). This interpretation may also help explain the minimal effects reported for ACC lesions on object and location recognition memory in rodents (Ennaceur et al., 1997). To our knowledge, no rodent lesion study has looked at remote object memory representations, but it is likely that object/place associations are no different from other memories: initially they are hippocampus dependent and then are transferred to cortex over time (i.e., consolidated) for long-term storage. Admittedly, our $\mathrm{R}$-Exp/S-Exp comparison does not address whether the passage of time ( 2 weeks, on average) or the amount of experience with the objects (seven re-exposure sessions) was the crucial component of the familiarization process. However, identification of comparable correlates $30 \mathrm{~d}$ following familiarization provided the clear evidence that the ACC is in fact either a site for consolidation or a location to which a remote memory is retrieved for on-line comparison with incoming sensory information.

Previous studies have implicated the ACC in remote memory retrieval. These studies provide compelling evidence for the transfer of task-critical information from the hippocampus to the
ACC, as assayed typically $1 \mathrm{~d}$ or 1 month or more after learning (Frankland et al., 2004; Teixeira et al., 2006; Ding et al., 2008; Restivo et al., 2009; Vetere et al., 2011). In a more recent study, Vetere et al. (2011) demonstrated a robust learning-related increase in spine density in caudal ACC layers II/III within the first $8 \mathrm{~d}$ after training. Inhibiting spinogenesis during this time disrupted subsequent expression of fear memory. Their study clearly reveals that consolidation-associated structural changes begin very shortly after training and develop along a time course almost precisely matching the familiarization protocol used in the present study. Our data, along with those of Vetere et al. (2011), indicate the gradual nature of consolidation and the stabilization of memories over time.

It is worth considering other alternative hypotheses here. One alternative involves the role of the ACC in premotor planning. In primates, neurons in the cingulate motor areas (Picard and Strick, 1996; Dum and Strick, 2002) exhibit activity associated with simple movements (Kollias et al., 2001; Dum and Strick, 2002) as well as the integration of sensory information and the execution of task-related behaviors (Isomura et al., 2003; Beudel and de Jong, 2009). Cingulate activity could reflect movements involved in exploration of objects and locations, but this hypothesis fails to account for our most interesting correlates. First, despite behaving indistinguishably from S-Exp mice, only extensively familiarized R-Exp mice exhibited neuronal responses to the absent object. Second, over half of these responses developed only after the object had been removed (starts-to-absent responses). Third, 37\% of R-Exp neuron absent-object responses exhibited delayed onsets, independent of changes in exploratory behavior, which is certainly inconsistent with a strict sensorimotor hypothesis. Both the behavioral response and that of other simultaneously recorded neurons is immediately evident, so it is not a global state change or that the animal did not notice the change. The delayed onset may instead reflect the inherent instability of a representation during consolidation, or perhaps the time it takes to make a new, updated representation of the context that takes the object's removal into account.

Another alternative is the response by Rudy et al. (2005) to the proposed role of the ACC in recall of remotely learned information. This alternative draws upon the role of the prefrontal cortex in cognitive control and top-down attention (O'Reilly, 1999; Miller and Cohen, 2001). Briefly, they hypothesize that weakened memory traces may be responsible for the increased ACC activation observed by Bontempi et al. (1999) and Frankland et al. (2004). In their view, the more remote the memory, the greater the top-down control required for effective recall. This is not applicable to our data as both R-Exp an S-Exp mice had last seen both objects equally recently. However, their suggestion that conflict detectors may be involved is worth considering in light of our starts-to-absent responses. According to conflict monitoring theory, situations involving errors or requiring the inhibition of prepotent responses create conflict and engage the ACC (Botvinick et al., 2001; Botvinick, 2007). In the present study, the high degree of familiarity of R-Exp mice to the objects would establish a strong expectation of how the environment and the elements within it should appear, effectively establishing a set of prepotent responses to the environment. The starts-to-absent response could therefore represent the disconnect between incoming sensory data and the representation retrieved from memory, as might be predicted from the extensive literature on error-related signals in the anterior cingulate (van Veen and Carter, 2006). There is, admittedly, a gulf between innate behaviors in a mouse and the complex behavioral tasks used with humans and nonhu- 
man primates to study neural correlates of conflict monitoring (Botvinick, 2007). But the starts-to-absent response is qualitatively distinct from the persistent activity represented by maintained-to-absent cells, and as such represents a distinct class of response. Regardless, this interpretation still invokes a mnemonic representation of the objects and the environment in the caudal ACC: one cannot have errors without expectations.

Thus, object correlates exhibited by ACC neurons exhibit features predicted for memories in the process of consolidation: they are initially labile, stabilize over time, and last indefinitely. Familiarization engenders neuronal responses to object removal. These include maintained-to-absent responses, representing a persistence of activity to an object that is no longer present, and startsto-absent responses, which are evoked directly by that object's removal. Remarkably, absent-object correlates are still seen 1 month following familiarization. These data demonstrate that the caudal ACC, along with the hippocampus and medial prefrontal, lateral entorhinal, and perirhinal cortices, is involved in conveying object/place associations (Barker and Warburton, 2008; Komorowski et al., 2009; Deshmukh and Knierim, 2011). More broadly, our data may provide part of the substrate for schema-dependent learning. Tse et al. (2007) describe this phenomenon in which prior knowledge facilitates rapid systemslevel consolidation. In their model, cortical structures including the caudal ACC successfully mediate the rapid acquisition of new paired-associates when placed against the backdrop of prior experience (Tse et al., 2011). In other words, the object/place associations strengthen over time as the animal becomes familiar with the features of the task and the environment. Our data clearly demonstrate the strengthening of neural correlates for object/ place associations with familiarization, potentially revealing the backdrop on which new paired-associates are encoded. In summary, our results strongly suggest electrophysiological correlates of consolidated memory in neurons of the ACC, describing (1) how familiarity stabilizes representations in the ACC, (2) how memory for some elements of that representation are reflected at the level of individual ACC neurons, and (3) how a specific set of neurons reflect a discontinuity between the representation retrieved from memory and the real world.

\section{References}

Barker GR, Warburton EC (2008) NMDA receptor plasticity in the perirhinal and prefrontal cortices is crucial for the acquisition of long-term object-in-place associative memory. J Neurosci 28:2837-2844.

Berg RW, Kleinfeld D (2003) Rhythmic whisking by rat: retraction as well as protraction of the vibrissae is under active muscular control. J Neurophysiol 89:104-117.

Beudel M, de Jong BM (2009) Overlap and segregation in predorsal premotor cortex activations related to free selection of self-referenced and target-based finger movements. Cereb Cortex 19:2361-2371.

Bontempi B, Laurent-Demir C, Destrade C, Jaffard R (1999) Timedependent reorganization of brain circuitry underlying long-term memory storage. Nature 400:671-675.

Botvinick MM (2007) Conflict monitoring and decision making: reconciling two perspectives on anterior cingulate function. Cogn Affect Behav Neurosci 7:356-366.

Botvinick MM, Braver TS, Barch DM, Carter CS, Cohen JD (2001) Conflict monitoring and cognitive control. Psychol Rev 108:624-652.

Brecht M, Krauss A, Muhammad S, Sinai-Esfahani L, Bellanca S, Margrie TW (2004) Organization of rat vibrissa motor cortex and adjacent areas according to cytoarchitectonics, microstimulation, and intracellular stimulation of identified cells. J Comp Neurol 479:360-373.

Curtis CE, Lee D (2010) Beyond working memory: the role of persistent activity in decision making. Trends Cogn Sci 14:216-222.

Deshmukh SS, Knierim JJ (2011) Representation of non-spatial and spatial information in the lateral entorhinal cortex. Front Behav Neurosci 5:69.
Ding HK, Teixeira CM, Frankland PW (2008) Inactivation of the anterior cingulate cortex blocks expression of remote, but not recent, conditioned taste aversion memory. Learn Mem 15:290-293.

Dudai Y, Morris RG (2000) To consolidate or not to consolidate: what are the questions? In: In brain, perception, and memory. Advances in cognitive sciences (Bolhius J, ed), pp 149-162. Oxford, UK: Oxford UP.

Dum RP, Strick PL (2002) Motor areas in the frontal lobe of the primate. Physiol Behav 77:677-682.

Ennaceur A, Neave N, Aggleton JP (1997) Spontaneous object recognition and object location memory in rats: the effects of lesions in the cingulate cortices, the medial prefrontal cortex, the cingulum bundle and the fornix. Exp Brain Res 113:509-519.

Frankland PW, Bontempi B (2005) The organization of recent and remote memories. Nat Rev Neurosci 6:119-130.

Frankland PW, Bontempi B, Talton LE, Kaczmarek L, Silva AJ (2004) The involvement of the anterior cingulate cortex in remote contextual fear memory. Science 304:881-883.

Fyhn M, Molden S, Witter MP, Moser EI, Moser MB (2004) Spatial representation in the entorhinal cortex. Science 305:1258-1264.

Gray CM, Maldonado PE, Wilson M, McNaughton B (1995) Tetrodes markedly improve the reliability and yield of multiple single-unit isolation from multi-unit recordings in cat striate cortex. J Neurosci Methods 63:43-54.

Hammond RS, Tull LE, Stackman RW (2004) On the delay-dependent involvement of the hippocampus in object recognition memory. Neurobiol Learn Mem 82:26-34.

Han CJ, O'Tuathaigh CM, van Trigt L, Quinn JJ, Fanselow MS, Mongeau R, Koch C, Anderson DJ (2003) Trace but not delay fear conditioning requires attention and the anterior cingulate cortex. Proc Natl Acad Sci U S A 100:13087-13092.

Isomura Y, Ito Y, Akazawa T, Nambu A, Takada M (2003) Neural coding of "attention for action" and "response selection" in primate anterior cingulate cortex. J Neurosci 23:8002-8012.

Jones BF, Groenewegen HJ, Witter MP (2005) Intrinsic connections of the cingulate cortex in the rat suggest the existence of multiple functionally segregated networks. Neuroscience 133:193-207.

Kollias SS, Alkadhi H, Jaermann T, Crelier G, Hepp-Reymond MC (2001) Identification of multiple nonprimary motor cortical areas with simple movements. Brain Res Brain Res Rev 36:185-195.

Komorowski RW, Manns JR, Eichenbaum H (2009) Robust conjunctive item-place coding by hippocampal neurons parallels learning what happens where. J Neurosci 29:9918-9929.

Lechner HA, Squire LR, Byrne JH (1999) 100 years of consolidation-remembering Muller and Pilzecker. Learn Mem 6:77-87.

Markus EJ, Barnes CA, McNaughton BL, Gladden VL, Skaggs WE (1994) Spatial information content and reliability of hippocampal CA1 neurons: effects of visual input. Hippocampus 4:410-421.

Marr D (1970) A theory for cerebral neocortex. Proc R Soc Lond B Biol Sci 176:161-234

Marr D (1971) Simple memory: a theory for archicortex. Philos Trans R Soc Lond B Biol Sci 262:23-81.

Miller EK, Cohen JD (2001) An integrative theory of prefrontal cortex function. Annu Rev Neurosci 24:167-202.

Muller RU, Kubie JL (1987) The effects of changes in the environment on the spatial firing of hippocampal complex-spike cells. J Neurosci 7:1951-1968.

O'Keefe J, Dostrovsky J (1971) The hippocampus as a spatial map. Preliminary evidence from unit activity in the freely-moving rat. Brain Res 34:171-175.

O'Reilly MF (1999) Effects of presession attention on the frequency of attention-maintained behavior. J Appl Behav Anal 32:371-374.

Picard N, Strick PL (1996) Motor areas of the medial wall: a review of their location and functional activation. Cereb Cortex 6:342-353.

Restivo L, Vetere G, Bontempi B, Ammassari-Teule M (2009) The formation of recent and remote memory is associated with time-dependent formation of dendritic spines in the hippocampus and anterior cingulate cortex. J Neurosci 29:8206-8214.

Rivard B, Li Y, Lenck-Santini PP, Poucet B, Muller RU (2004) Representation of objects in space by two classes of hippocampal pyramidal cells. J Gen Physiol 124:9-25. 
Rudy JW, Biedenkapp JC, O’Reilly RC (2005) Prefrontal cortex and the organization of recent and remote memories: an alternative view. Learn Mem 12:445-446.

Sarkisyan G, Hedlund PB (2009) The 5-HT7 receptor is involved in allocentric spatial memory information processing. Behav Brain Res 202:26-31.

Scoville WB, Milner B (1957) Loss of recent memory after bilateral hippocampal lesions. J Neurol Neurosurg Psychiatry 20:11-21.

Squire LR, Alvarez P (1995) Retrograde amnesia and memory consolidation: a neurobiological perspective. Curr Opin Neurobiol 5:169-177.

Squire LR, Bayley PJ (2007) The neuroscience of remote memory. Curr Opin Neurobiol 17:185-196.

Teixeira CM, Pomedli SR, Maei HR, Kee N, Frankland PW (2006) Involvement of the anterior cingulate cortex in the expression of remote spatial memory. J Neurosci 26:7555-7564.

Tse D, Langston RF, Kakeyama M, Bethus I, Spooner PA, Wood ER, Witter MP, Morris RG (2007) Schemas and memory consolidation. Science 316:76-82.

Tse D, Takeuchi T, Kakeyama M, Kajii Y, Okuno H, Tohyama C, Bito H,
Morris RG (2011) Schema-dependent gene activation and memory encoding in neocortex. Science 333:891-895.

van Veen V, Carter CS (2006) Error detection, correction, and prevention in the brain: a brief review of data and theories. Clin EEG Neurosci 37:330-335.

Vetere G, Restivo L, Cole CJ, Ross PJ, Ammassari-Teule M, Josselyn SA, Frankland PW (2011) Spine growth in the anterior cingulate cortex is necessary for the consolidation of contextual fear memory. Proc Natl Acad Sci U S A 108:8456-8460.

Weible AP, Rowland DC, Pang R, Kentros C (2009) Neural correlates of novel object and novel location recognition behavior in the mouse anterior cingulate cortex. J Neurophysiol 102:2055-2068.

Wirtshafter D (2005) Cholinergic involvement in the cortical and hippocampal Fos expression induced in the rat by placement in a novel environment. Brain Res 1051:57-65.

Xu H, Wu LJ, Wang H, Zhang X, Vadakkan KI, Kim SS, Steenland HW, Zhuo M (2008) Presynaptic and postsynaptic amplifications of neuropathic pain in the anterior cingulate cortex. J Neurosci 28:7445-7453. 